\title{
Reply to the commentary "To Gorelenkova Miller and Mieyal (2015): Sulfhydryl-mediated redox signaling in inflammation: role in neurodegenerative diseases" by Mieyal JJ
}

\author{
Masashi Kato $^{1,2} \cdot$ Hiromasa Ninomiya $^{1,2} \cdot$ Masao Maeda $^{1,2} \cdot$ Cimi Ilmiawati $^{1,2}$. \\ M M A. Al Hossain ${ }^{1}$ Masafumi Yoshinaga ${ }^{1,2} \cdot$ Nobutaka Ohgami $^{1,2}$
}

Received: 3 March 2016 / Accepted: 4 April 2016 / Published online: 15 April 2016

(C) Springer-Verlag Berlin Heidelberg 2016

Our comment promoted by a "Reply to the Commentary To Gorelenkova Miller and Mieyal (2015): Sulfhydrylmediated redox signaling in inflammation: role in neurodegenerative diseases by M. Kato" by Dr. Mieyal (2016). Dr Mieyal suggests to discern pro- and anti-apoptotic effects of RET dimerization and to clarify the regulatory mechanisms through focusing on the reversible sulfhydryl adducts. Since these are very important issues, we will reply to them following our previous results for ligand binding-independent signaling pathways developed by oxidative stresses with focus on RET, a key player for neurodegenerative diseases (Nakashima et al. 2002).

RET, a receptor tyrosine kinase, mainly contributes to the development and maintenance of neurons, though RET-mediated promotion of apoptosis has also been reported (Bordeaux et al. 2000; Kato et al. 2002; Asai et al. 2006). RET is activated by the glial cell line-derived neurotrophic factor (GDNF) family of ligands including neurturin, artemin and persephin (Asai et al. 2006). One of the characteristics of the ligand-mediated RET kinase activation is the requirement of GDNF family receptors (GFRs) alpha1-4 (Asai et al. 2006; Bespalov and Saarma 2007). The formation of a GDNF-mediated heterotetramer consisting of dimerized RET and dimerized

Masashi Kato

katomasa@med.nagoya-u.ac.jp

1 Department of Occupational and Environmental Health, Nagoya University Graduate School of Medicine, 65 Tsurumai-cho, Showa-ku, Nagoya, Aichi 466-8550, Japan

2 Voluntary Body for International Health Care in Universities, 65 Tsurumai-cho, Showa-ku, Nagoya, Aichi 466-8550, Japan
GFR alpha1 is essential for RET-mediated growth of neural cells (Sariola and Saarma 1999; Bespalov and Saarma 2007). Our previous studies showed that oxidative stresses promote activation of RET kinase with dimerized RET protein via disulfide bond formation (Kato et al. 2016). To our knowledge, however, it remains unclear whether oxidative stresses promote dimerization of GFRs via disulfide bond formation. We assume that the three-dimensional structure of the heterotetramer induced by the ligands is different from that induced by oxidative stresses because it has been shown in previous studies that oxidative stresses for RET indifferently promote formation of a polymer as well as a dimer (Akhand et al. 1999; Kato et al. 2000). Clarification of the effects of oxidative stresses on three-dimensional structures of the heterotetramer may be important to elucidate the regulatory mechanisms for RET via reversible sulfhydryl adducts.

Despite the fact that both stimulations of ligands and oxidative stresses promote activity and dimerization of RET, ligands and oxidative stresses promote survival and death, respectively, in neural cells (Nakashima et al. 2002). The results of our previous study showed that oxidative stresses indifferently promote dimerization of various protein kinases in addition to RET on the cell surface through modification of cysteine sulfhydryl (SH) groups (Nakashima et al. 2002). Different signals based on different specificities for dimer formation of RET between ligands and oxidative stresses may cause the opposite effects on RET dimerization. Further analysis of the biological significance and mechanisms of ligand-mediated and oxidative stress-mediated dimerization of RET may be useful to clarify the role of sulfhydryl-mediated redox signaling in neurodegenerative diseases. 


\section{References}

Akhand AA, Kato M, Suzuki H et al (1999) Carbonyl compounds cross-link cellular proteins and activate protein-tyrosine kinase p60c-Src. J Cell Biochem 72(1):1-7

Asai N, Fukuda T, Wu Z et al (2006) Targeted mutation of serine 697 in the Ret tyrosine kinase causes migration defect of enteric neural crest cells. Development 133(22):4507-4516

Bespalov MM, Saarma M (2007) GDNF family receptor complexes are emerging drug targets. Trends Pharmacol Sci 28(2):68-74

Bordeaux MC, Forcet C, Granger L et al (2000) The RET proto-oncogene induces apoptosis: a novel mechanism for Hirschsprung disease. EMBO J 19(15):4056-4063

Kato M, Iwashita T, Akhand AA et al (2000) Molecular mechanism of activation and superactivation of Ret tyrosine kinases by ultraviolet light irradiation. Antioxid Redox Signal 2(4):841-849

Kato M, Takeda K, Kawamoto Y et al (2002) Repair by Src kinase of function-impaired RET with multiple endocrine neoplasia type $2 \mathrm{~A}$ mutation with substitutions of tyrosines in the $\mathrm{COOH}-$ terminal kinase domain for phenylalanine. Cancer Res 62(8):2414-2422

Kato M, Ninomiya H, Maeda M et al (2016) Commentary To Gorelenkova Miller and Mieyal (2015): sulfhydryl-mediated redox signaling in inflammation: role in neurodegenerative diseases. Arch Toxicol 90(4):1017-1018

Mieyal JJ (2016) Reply to the Commentary to Gorelenkova Miller and Mieyal (2015): sulfhydryl-mediated redox signaling in inflammation: role in neurodegenerative diseases by M. Kato. Arch Toxicol 90(4):1019-1020

Nakashima I, Kato M, Akhand AA et al (2002) Redox-linked signal transduction pathways for protein tyrosine kinase activation. Antioxid Redox Signal 4(3):517-531

Sariola H, Saarma M (1999) GDNF and its receptors in the regulation of the ureteric branching. Int J Dev Biol 43(5):413-418 